\title{
Echo Location Based Bat Algorithm for Energy Efficient WSN Routing
}

\author{
Anwer Mustafa Hilal ${ }^{1, *}$, Siwar Ben Haj Hassine ${ }^{2}$, Jaber S. Alzahrani ${ }^{3}$, Masoud Alajmi ${ }^{4}$, \\ Fahd N. Al-Wesabi ${ }^{2,5}$, Mesfer Al Duhayyim ${ }^{6}$, Ishfaq Yaseen $^{1}$ and Abdelwahed Motwakel ${ }^{1}$
}

\author{
${ }^{1}$ Department of Computer and Self Development, Preparatory Year Deanship, Prince Sattam bin Abdulaziz University, \\ AlKharj, Saudi Arabia \\ ${ }^{2}$ Department of Computer Science, College of Science and Arts, King Khalid University, Mahayil Asir, Saudi Arabia \\ ${ }^{3}$ Department of Industrial Engineering, College of Engineering at Alqunfudah, Umm Al-Qura University, Saudi Arabia \\ ${ }^{4}$ Department of Computer Engineering, College of Computers and Information Technology, Taif University, Taif, 21944, \\ Saudi Arabia \\ ${ }^{5}$ Faculty of Computer and IT, Sana'a University, Sana'a, Yemen \\ ${ }^{6}$ Department of Natural and Applied Sciences, College of Community-Aflaj, Prince Sattam bin Abdulaziz University, \\ Saudi Arabia \\ *Corresponding Author: Anwer Mustafa Hilal. Email: a.hilal@psau.edu.sa \\ Received: 19 October 2021; Accepted: 16 December 2021
}

\begin{abstract}
Due to the wide range of applications, Wireless Sensor Networks (WSN) are increased in day to day life and becomes popular. WSN has marked its importance in both practical and research domains. Energy is the most significant resource, the important challenge in WSN is to extend its lifetime. The energy reduction is a key to extend the network's lifetime. Clustering of sensor nodes is one of the well-known and proved methods for achieving scalable and energy conserving WSN. In this paper, an energy efficient protocol is proposed using metaheuristic Echo location-based BAT algorithm (ECHO-BAT). ECHO-BAT works in two stages. First Stage clusters the sensor nodes and identifies tentative Cluster Head $(\mathrm{CH})$ along with the entropy value using BAT algorithm. The second stage aims to find the nodes if any, with high residual energy within each cluster. $\mathrm{CHs}$ will be replaced by the member node with high residual energy with an objective to choose the $\mathrm{CH}$ with high energy to prolong the network's lifetime. The performance of the proposed work is compared with Low-Energy Adaptive Clustering Hierarchy (LEACH), Power-Efficient Zoning Clustering Algorithm (PEZCA) and Chaotic Firefly Algorithm $\mathrm{CH}(\mathrm{CFACH})$ in terms of lifetime of network, death of first nodes, death of 125 th node, death of the last node, network throughput and execution time. Simulation results show that ECHO-BAT outperforms the other methods in all the considered measures. The overall delivery ratio has also significantly optimized and improved by approximately $8 \%$, proving the proposed approach to be an energy efficient WSN.
\end{abstract}

Keywords: Wireless sensor networks; BAT algorithm; energy efficient; clustering; cluster head; energy consumption 


\section{Introduction}

The rapid evolution of wireless technology and growth in microelectronics has led to the massive increase in wireless sensor networks. Initially, WSNs were designed and developed for military operations, but now it is applied in almost all domains ranging from health to entertainment [1,2]. WSN is an infrastructure-less network technology with a large number of sensor nodes in an ad-hoc manner to monitor the system. WSN may contain hundreds or even thousands of sensor nodes, which is a low power constrained device. It is composed of low processing power processor, less memory, a sensor board, a battery and a radio for wireless communication. Sensor node size ranges from the size of a grain of dust in size of a shoe box. Depending on the functional parameters like energy consumption, computational speed rate bandwidth and memory, the cost of the sensor nodes may vary from few hundreds to lakh. A sensor node monitors the processing and storage unit for analyzing information using their sensor modules [3]. The main issues in WSN are high energy consumption for data transmission, demand for high bandwidth, Quality of service, data processing and compression technique and network lifetime. Network topology and energy consumption are two significant factors to be considered in WSN. These networks classify using the energy efficient hierarchical unequal clustering [4] probabilistic neural network [5], Neuro fuzzy approach [6] and artificial intelligencebased intrusion detection system [7].

Due to limited computation, limited energy, limited memory and storage [8] data traffic in the network should be minimized. This can be achieved by grouping the sensor nodes into clusters as it has advantages in scalability, energy saving, resource sharing and efficient resource allocation. A cluster has a group of sensor nodes with a similarity measure along with a cluster head. $\mathrm{CH}$ coordinates the sensor nodes associates with other $\mathrm{CHs}$ with an aim to send the data to the sink node for Base Station (BS).

Major impact in the network is to enhance the lifetime of the network. Routing establishes a path to send data from the cluster to the base station to select energy efficient WSN. WSNs are classified as homogeneous or heterogeneous according to their energy emission levels of sensor nodes. When all the nodes have an equal amount of energy utilization, it is said to be homogeneous while heterogeneous networks have more initial energy supplied to a few sensor nodes in comparison to the usual nodes $[9,10]$. Selection of $\mathrm{CH}$ in sensor network is a NP problem as energy balanced optimal data aggregation cannot take place in a polynomial time [11]. Thus, meta-heuristic optimization algorithms are proved to be potent in facilitating efficient $\mathrm{CH}$ selection process [12].

This paper proposes an energy efficient method when transmitting and receiving data from sensor node and BS by reflecting the topology of the network which in turn increases the lifespan of the nodes and network lifetime. The proposed method involves two stages namely: Initialization stage and transmission stage. The first stage finds the tentative cluster head with the entropy value and finally optimal permanent cluster head is selected using echolocation behaviour of BAT algorithm. Transmission stage finds an optimal path from the cluster to the base station.

The paper is composed in following manner. Section 2 reviews the literature of the existing schemes. Section 3 describes the proposed scheme to reduce sensor nodes energy consumption. Performance of the proposed scheme is evaluated through simulation. This work is concluded with future research directions in Section 5. 


\section{Literature Review}

Research in WSNs can be classified into two methods: 1. To improve the performance of the network by improving the performance the most important component, sensor nodes. 2 . To improve the network lifetime by reducing the consumption of energy while transmitting and receiving data. Clustering the sensor nodes is performed based on characteristics of clusters, clustering strategy and potency of CHs. This research work aims to stabilise the cluster formation and finds an optimal path to transmit and receive data from and to the clusters and base station. The proceeding section aims to review the existing methods already developed to develop energy efficient network model. Finally, it studies the methods to find the route between the clusters and base station to transmit and receive data.

Gong et al. [13] devised a distributed energy efficient clustering (DEEC) for heterogeneous WSNs. The authors used the ratio between average energy of the network and residual energy of each node to identify $\mathrm{CH}$. Based on the initial and residual energy of the nodes, the nodes with high initial and residual energy are eligible to become $\mathrm{CH}$ s compared to the nodes with less energy. The results prove that DEEEC could send eminent information when compared to the current clustering methods used in heterogeneous environments attains prolonged lifetime.

Kuila et al. [14] formulated a design which was suitable for novel clustering the methodology of EECS for wireless sensor networks, which is adaptable for timely information database applications. During the time of fetching good $\mathrm{CH}$ distribution, the EECS system chooses $\mathrm{CH}$ with large residual energy following the local radio communication. Qing et al. [15] formulated a novel method called Distributed Energy-Efficient Clustering having Improved Coverage (DEECIC) algorithm. Based on local information DEECIC targets in assigning a unique ID to every node and also clusters the minimum quantity of $\mathrm{CHs}$ to surround the complete network.

Ye et al. in his paper [16] associated voting method and clustering algorithm to develop a new clustering scheme for secure localization of sensor networks. Clustering algorithm based on minimum spanning tree-PSO of the weighted graph of the WSNs is proposed by Liu et al. [17]. Based on the energy consumption, the optimal route between the nodes and its cluster heads are found in the entire tree.

A distributed clustering algorithm for WSN is proposed in [18] by Yang et al., called multiobjective fuzzy clustering algorithm (MOFCA). Use residual energy, distance to sink, and node density to compute the competition radius of tentative $\mathrm{CH}$. If there exist any competitive $\mathrm{CH}$, it withdraws from $\mathrm{CH}$ competition. MOFCA seems to be effective and proved to be significant in extending the lifetime of the network.

Cao et al. [19] devised an energy aware clustering protocol using fuzzy logic (ECPF) for WSN. ECPF involves two phases namely: setup phase and steady state phase. The first phase elects the $\mathrm{CH}$ and forms the cluster. Frame generation and data collection will happen in steady state phase. Sert et al. in his work [20] analyzed the conditions necessary for increasing the energy efficiency of the nodes constituting a sensor network using a clustering routing technique and a location-based routing technique.

Authors proposed a WSN configuration method that increases the energy efficiency of each node and proved by experimental methods that the results were optimal. Taheri et al. [21] analyzed the problem of energy consumption of the single-hop and multi-hop transmissions in a single cluster. They determined a critical value of cluster area. MS-LEACH is based on the critical value and it outperforms $200 \%$ in terms of network life time. 


\section{Proposed ECHO-BAT Based Energy Efficient WSN Method}

Echolocation based BAT algorithm (ECHO-BAT) is proposed to develop an energy efficient WSN. Two stages are involved in the proposed scheme. The first stage is the initialization stage and the second stage is transmission stage. First step in stage 1 is to cluster the sensor nodes and chooses a tentative cluster head with its entropy value. Entropy value is obtained using the correlative measure of residual and original energy. Second step of stage 1 aims to find if there are any other nodes in the cluster with high residual energy. If it is found, then the tentative $\mathrm{CH}$ is replaced by the newly found node. Second step is achieved by employing BAT algorithm. Thus, the two steps in stage 1 results in choosing optimal CHs. Transmission stage establishes a path to reach the Base Station (BS). The overall architecture of the proposed method is represented in Fig. 1.

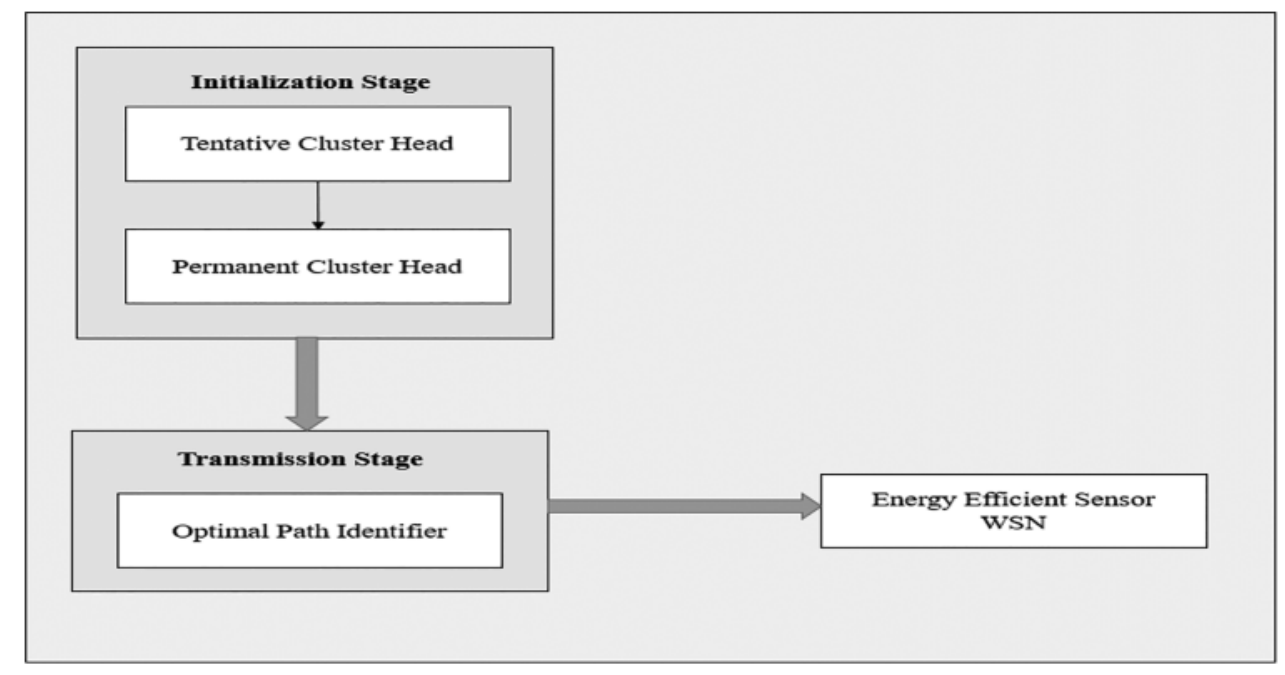

Figure 1: Overall architecture of proposed ECHO-BAT

\subsection{Initialization Stage}

Initialization stage involves two steps namely: Tentative $\mathrm{CH}$ selection step as used in [22] and Permanent $\mathrm{CH}$ selection steps.

\subsubsection{Tentative CH Selection Step}

The rotating epoch for $\mathrm{CHs}$ to [1/optcl]. The rotating epoch and the reasons for deploying HWSNs become ineligible. All the sensor nodes should finalize its own selection entropy by considering its initial and residual energy level. Every computation of energy outlined in this method is the variable quantity. The arithmetic mean of this variable quantity is energy entropy. Entropy is found using Eq. (1).

$E N T_{i}=-\sum_{i=1}^{n} \times E n_{i}(r) \log _{b} E n_{\text {avg }}(r)$ 
$E n_{i}(r)$ is the residual energy of sensor node $S_{i}$ at round r. $E n_{\text {avg }}(r)$ is the average energy of the network in round $\mathrm{r}$.

$E n_{\text {avg }}(r)=\frac{1}{N} \sum E n_{i}(r)$

$E N T_{i}$ oscillate around optcl considering the direction of the two relative energy which helps sensor node to manage its entropy. All the nodes evaluate its probability ad chooses a random number between 0 and 1 . When the probability value is less than the condition the node chooses itself to be tentative $\mathrm{CH}$. Si's threshold of entropy is correlated with $E N T_{i}$ as

$$
\begin{gathered}
T h\left(S_{i}\right)=0, \text { if } S_{i} \text { does not belong to C, } \\
T h\left(S_{i}\right)=\left\{\frac{E N T_{i}}{1-E N T_{i}\left(\operatorname{rmod}\left[\frac{1}{E N T_{i}}\right]\right)} \text { if } S_{i} \in \mathrm{C}\right.
\end{gathered}
$$

Where $\mathrm{C}$ is the group of nodes, Even, in the final round if $S_{i}$ is not elected as $\mathrm{CH}$ then $S_{i}$ belongs to $\mathrm{C}$.

\subsubsection{Permanent CH Selection Step}

The bat algorithm is a meta heuristic search optimization algorithm in which all bats collaborate in populations to achieve a collective goal. Each bat is a solution in search space called a local solution. This is evaluated by calculating the fitness function and updates its position, velocity and frequency. Finally global solution is obtained after predefined number of iterations. The tentatively selected nodes will be replaced by nodes with high residual energy within the cluster. This is achieved by implementing BAT algorithm. The fitness function is calculated using Eq. (4). Which considers distance and energy?

Fitness $=\alpha \times f_{1}+(1-\alpha) \times f_{2}$

Where, a is a user defined constant, sub objectives are $f_{1}$ and $f_{2} . f_{1}$ is the sum of residual energy ratios of all nodes within a cluster to the residual energy of $\mathrm{CH}(\mathrm{k})$ and as per Eq. (5). The $f_{2}$ is the sum of inter cluster distance as in Eq. (6).

$f_{1}=\sum_{k=1}^{m} \frac{\sum_{j=1, j !=k}^{n_{c}} E(j)}{E(k)}$

Where $\mathrm{m}$ is the number of CHs. $n_{c}$ is the number of nodes in a cluster. $E(j)$ is the residual energy of node j. Remaining energy of $\mathrm{CH}(\mathrm{k})$ is $E(k)$.

$f_{2}=\sum_{k=1}^{m} \sum_{j=1, j \neq k}^{n_{c}} d(j, k)$

Where $\mathrm{d}(\mathrm{j}, \mathrm{k})$ is the distance between $\mathrm{CH}(\mathrm{k})$ and node $\mathrm{j}$.

The following are the steps employed by BAT algorithm to select permanent $\mathrm{CH}$. The flowchart of the BAT algorithm to choose appropriate $\mathrm{CH}$ is shown in Fig. 2. 


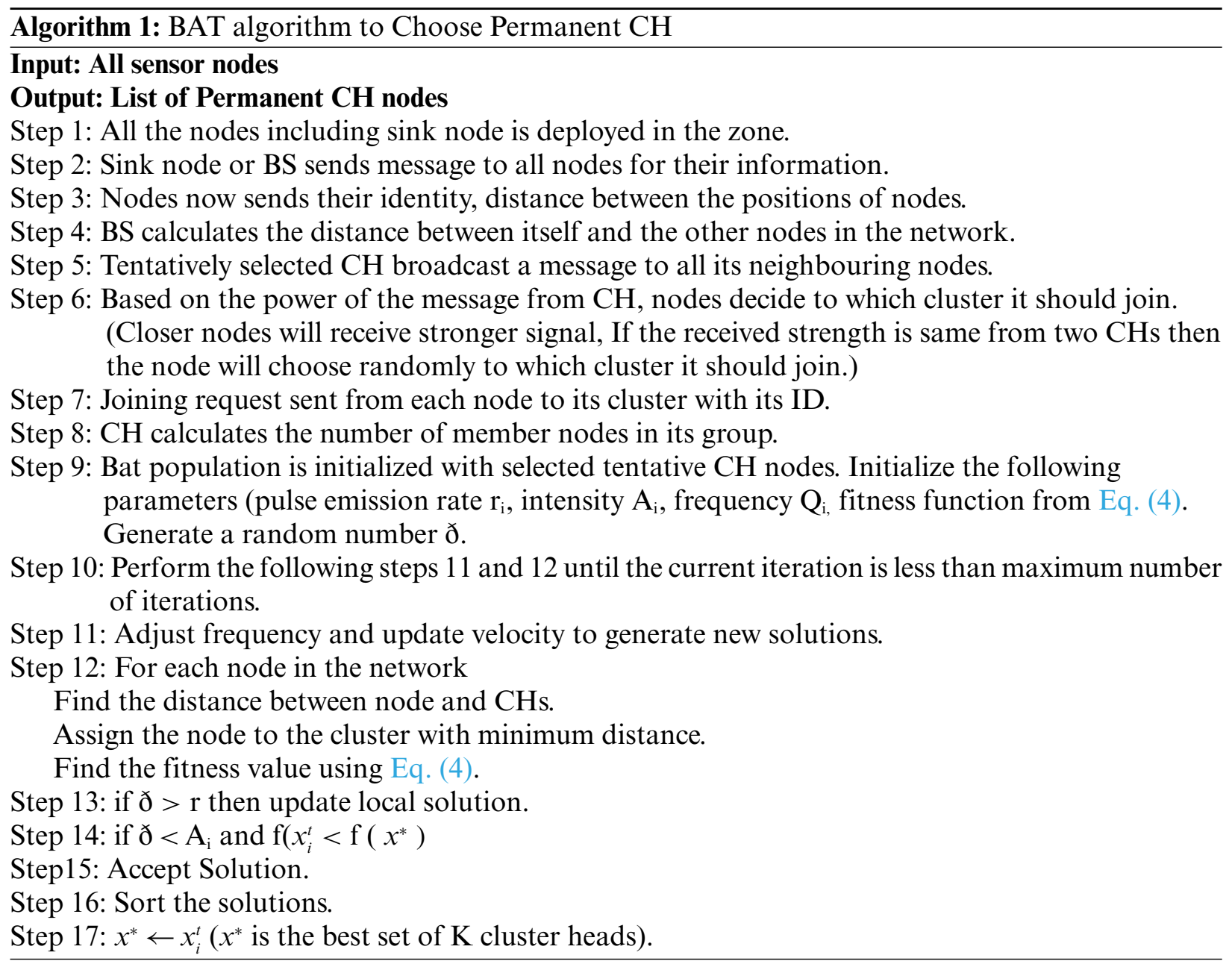

\subsection{Transmission Stage}

This stage deals data transmission. Based on the transmission distance data transmission from $\mathrm{Ch}$ to BS consumes energy. Considerably more energy will be consumed when the distance is large. Taking this into account, the next step after choosing the $\mathrm{CH}$ is to find an optimal path from each cluster to the BS. Dijkstra algorithm [23-25] is used to find the shortest path between $\mathrm{CH}$ and BS. The step involved in this process is shown in Algorithm 2.






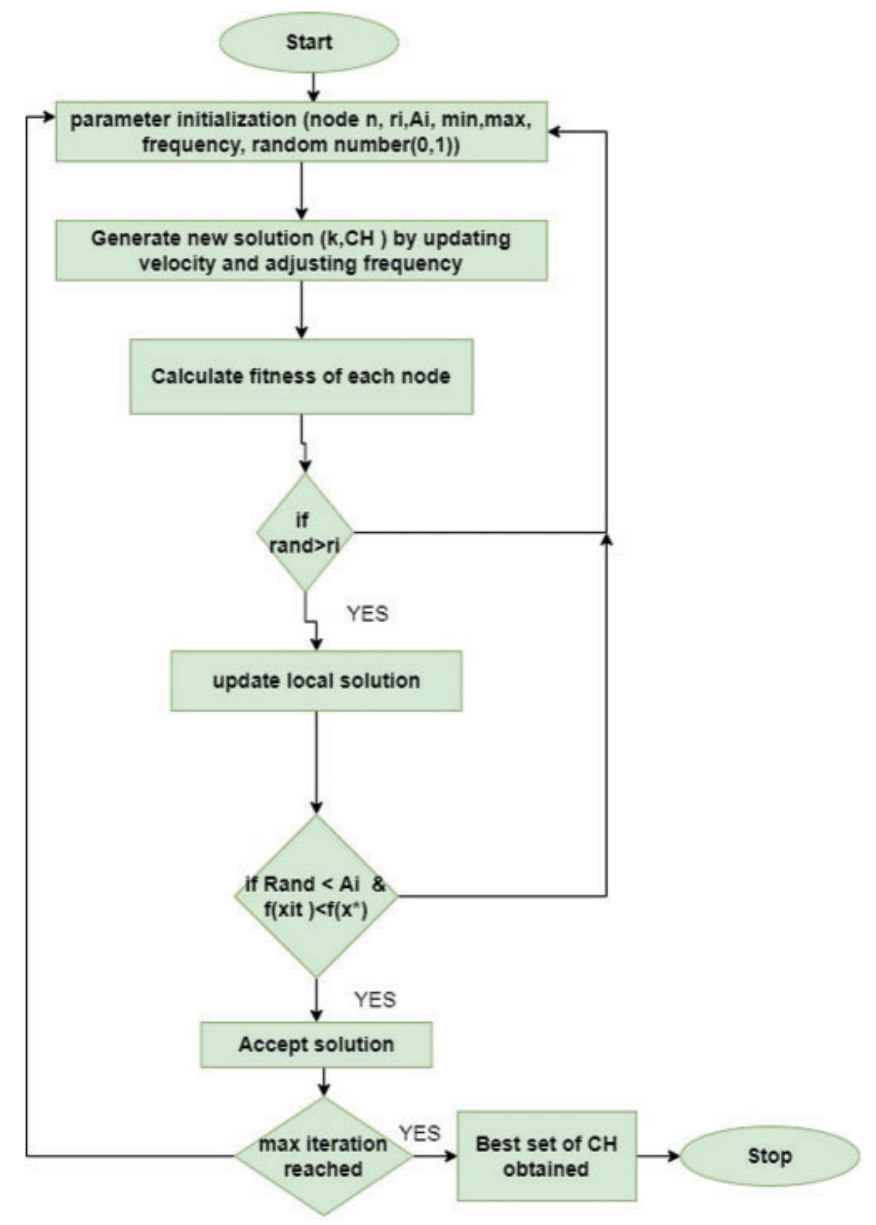

Figure 2: Flowchart of BAT to choose appropriate $\mathrm{CH}$

A node within each cluster sends the data to its $\mathrm{CH}$ and the $\mathrm{CH}$ collects and integrates the data received. $50 \%$ of data will be consumed in the network during this data transmission. The collected data will be sent to the BS which in-turn sends to the webserver to process the service.

\section{Simulation Results and Analysis}

This section analyses the results obtained by implementing ECHO-BAT in NS2 simulator. The performance is evaluated by comparing with LEACH, PEZCA and CFACH with five metrics namely: network lifetime, death of the first node, death of the 20th node, execution time and success rate. Setup parameters of the network are shown in Tab. 1. Initial energy of nodes from 0.5 to $0.8 \mathrm{~J}$ is considered and average results are considered. Network region of $100 \times 100 \mathrm{~m}^{3}$ is taken up with 250 nodes. The proposed and existing algorithms are run for 1000 rounds.

\subsection{Death of 1st, 125th Node, Last Node}

The network stability is calculated using the round in which the first node dies, round in which the last node dies. The number of rounds is fixed at 1000 with energy between 0.5 to $0.8 \mathrm{~J}$. The Figs. 3-6 
compares death of nodes $v$ s. number of rounds at which the first node dies for ECHO-BAT, LEACH, PEZCA and CFACH with the energy of $0.5,0.6,0.7$ and $0.8 \mathrm{~J}$.

Table 1: Simulation parameters

\begin{tabular}{ll}
\hline Parameters & Values \\
\hline Network area & $100 \times 100 \mathrm{~m}^{3}$ \\
Initial node energy & $0.5-0.8 \mathrm{~J}$ \\
Number of nodes & 1000 \\
Transmission range & $85 \mathrm{~m}$ \\
Data packet size & $500 \mathrm{bytes}$ \\
Energy consumption for sending data & $0.01 \mathrm{~J}$ \\
CH percentage & 0.05 \\
Packet size & $4000 \mathrm{bits}$ \\
\hline
\end{tabular}

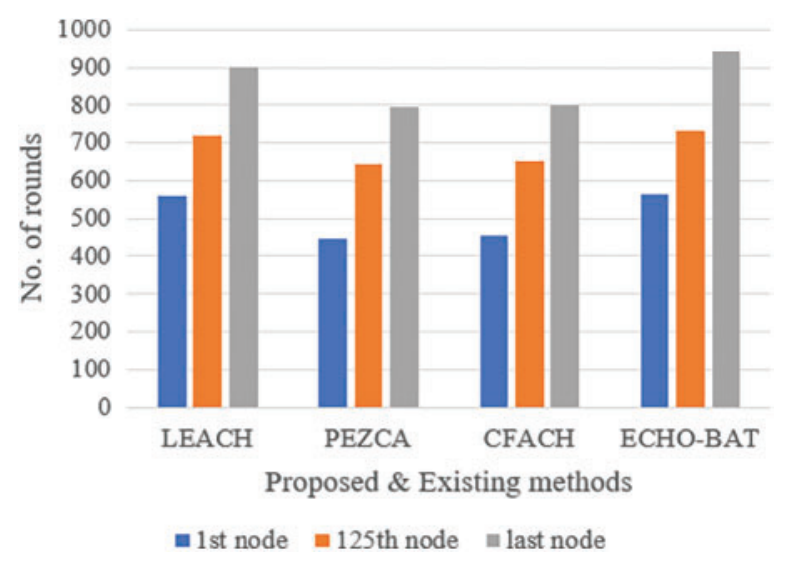

Figure 3: Node energy of $0.5 \mathrm{~J}$

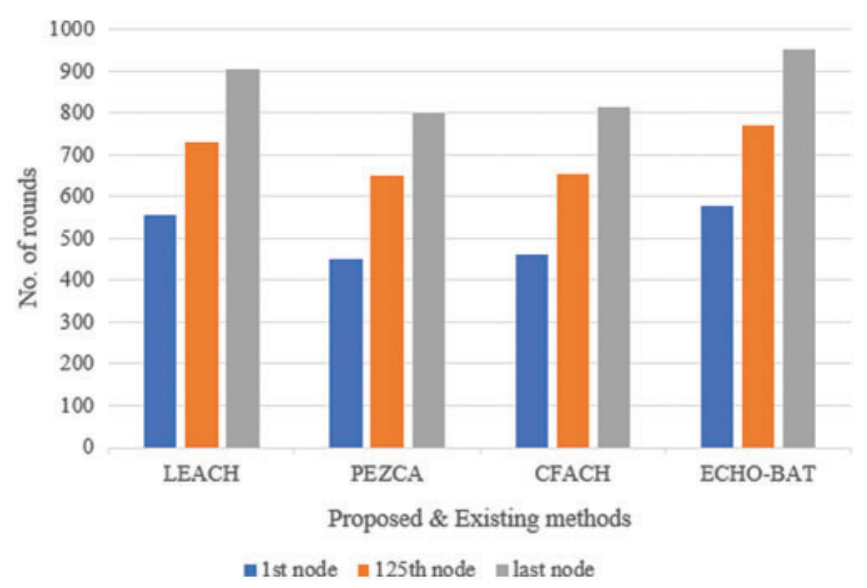

Figure 4: Node energy of $0.6 \mathrm{~J}$ 


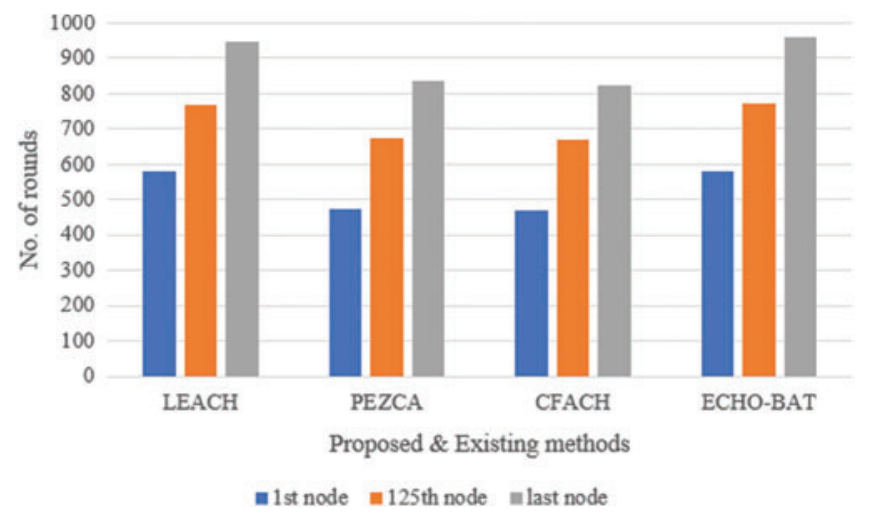

Figure 5: Node energy of $0.7 \mathrm{~J}$

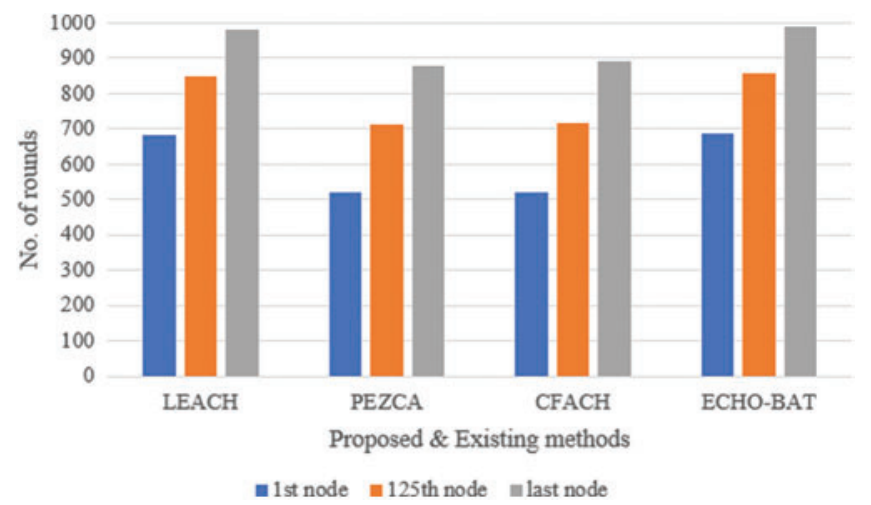

Figure 6: Node energy of $0.8 \mathrm{~J}$

Fig. 3 clearly shows that proposed ECHO-BAT shows higher network lifetime. In ECHO-BAT, first node dies at 564th round, 125 th node dies at 734 th round and last node dies only at 943rd round for $0.5 \mathrm{~J}$ energy. From this it is clear that lifetime of the network for ECHO-BAT is higher compared to LEACH, PEZCA and CFACH after finding the round at which first node, 125th node and last node dies. For $0.6 \mathrm{~J}$ of energy, first, 125th and last nodes die almost in the similar rounds in PEZCA and CFACH. But, LEACH and ECHO-BAT are stable in which lifetime of ECHO-BAT's network is superior than LEACH. This is evident from Fig. 4. For $0.7 \mathrm{~J}$ of energy, it is again proved from Fig. 5 that PECZA and CFACH are more or less similar like LEACH and ECHO-BAT. Fig. 6 shows PECZA outperformed CFACH for all three cases (first node death, $125^{\text {th }}$ node death and last node death). Whereas LEACH came much closer to ECHO-BAT for $0.8 \mathrm{~J}$ of energy.

\subsection{Network Throughput}

Throughput of the network is calculated based on the number of packets sent to BS for 50 nodes and 100 nodes at 1000 rounds. Network throughput is clearly evident from the Tab. 2. Given below for $0.5,0.6,0.7$ and $0.8 \mathrm{~J}$ sensor node energy.

It is observed from Tab. 2 that the number of packets sent to BS in LEACH, PECZ, CFACH and ECHO-BAT was found for 1000 rounds for 500 and 1000 sensor nodes. Tab. 2 shows the average results of the packets sent to BS for initial energy of $0.5 \mathrm{~J}$. ECHO-BAT sends 436237 packets on average, 
followed by LEACH with 413,760 packets as second and CFACH with 320358 packets as third and finally PECZA sends 284386 packets on average as least among the other three.

Table 2: Network throughput (500 nodes, 1000 nodes with $0.5 \mathrm{~J}$ initial energy)

\begin{tabular}{lll}
\hline Methods & 500 & 1000 \\
\hline LEACH & 272491 & 282538 \\
PECZA & 187654 & 193464 \\
CFACH & 210976 & 218764 \\
ECHO-BAT & 288265 & 298944 \\
\hline
\end{tabular}

Tab. 3 evidently shows that the proposed ECHO-BAT outperforms LEACH, PECZA and $\mathrm{CFACH}$ by sending more packets to BSC for 500 sensor nodes and 1000 sensor nodes. Network throughput for ECHO-BAT is 299864 and 301234 for 50 and 100 nodes respectively for $0.6 \mathrm{~J}$ of initial energy. LEACH seems to be closer to ECHO-BAT. PECZA and CFACH are almost similar for 0.6 J of initial energy.

Table 3: Network throughput (500 nodes, 1000 nodes with $0.6 \mathrm{~J}$ initial energy)

\begin{tabular}{lll}
\hline Methods & 500 & 1000 \\
\hline LEACH & 295631 & 297684 \\
PECZA & 228732 & 238765 \\
CFACH & 228865 & 238734 \\
ECHO-BAT & 299864 & 301234 \\
\hline
\end{tabular}

By considering the average of the number of packets sent from to BS, ECHO-BAT reaches the first position among LEACH, PECZ and CFACH for $0.7 \mathrm{~J}$ of energy. LEACH with second position and CFACH being the third and PECZA being the last.

It is evident from Tab. 4 that the network throughput in LEACH, PECZ, CFACH and ECHOBAT was found for 500 and 1000 nodes for 1000 rounds. The average number of packets sent to BS is clearly shown in Tab. 5 with ECHO-BAT sending highest number of packets for 500 as well as 1000 nodes. CFACH being the last with 237861 and 247714 for 500 and 1000 nodes respectively.

Table 4: Network throughput (500 nodes, 1000 nodes with $0.7 \mathrm{~J}$ initial energy)

\begin{tabular}{lll}
\hline Methods & 500 & 1000 \\
\hline LEACH & 295631 & 297684 \\
PECZA & 230021 & 238765 \\
CFACH & 228865 & 238734 \\
ECHO-BAT & 289864 & 301234 \\
\hline
\end{tabular}


Table 5: Network throughput (500 nodes, 1000 nodes with $0.8 \mathrm{~J}$ initial energy)

\begin{tabular}{lll}
\hline Methods & 500 & 1000 \\
\hline LEACH & 302345 & 315684 \\
PECZA & 244567 & 255775 \\
CFACH & 237861 & 247714 \\
ECHO-BAT & 309864 & 334534 \\
\hline
\end{tabular}

\subsection{Execution Time}

Execution time in seconds is considered as one of the important metrics in this work. Results are shown in terms of 200, 400, 600, 800 and 1000 nodes with respect to time in seconds for LEACH, PEZCA, CFACH and ECHO-BAT.

Fig. 7 shows the energy execution time of sensor nodes ranging from 200 to 1000 nodes for initial energy of $0.5 \mathrm{~J}$. It proves that ECHO-BAT takes minimum overall processing time followed by $\mathrm{LEACH}$, PEZCA and CFACH.

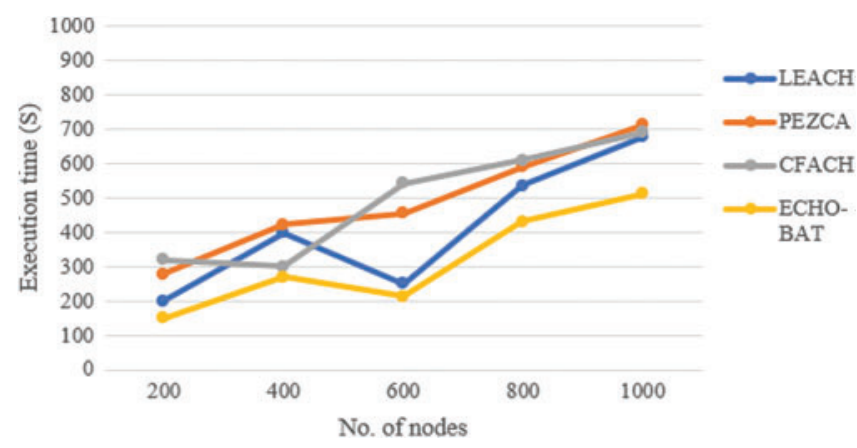

Figure 7: Execution time with $0.5 \mathrm{~J}$

Execution time for 200 to 1000 sensor nodes for $0.6 \mathrm{~J}$ of energy is displayed in Fig. 8. It is evident from the figure that proposed ECHO-BAT takes minimal time to process compared to other conventional methods namely LEACH, CFACH and PEZCA.

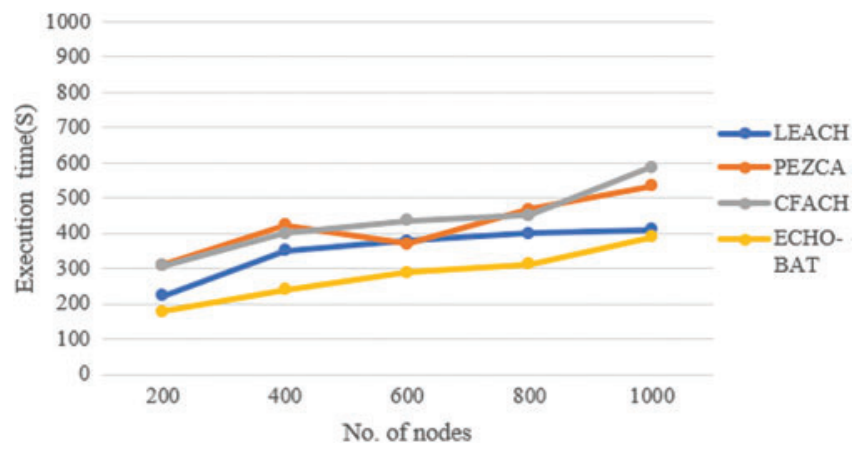

Figure 8: Execution time with $0.6 \mathrm{~J}$ 
Fig. 9 picturizes the processing time had taken by ECHO-BAT, LEACH, CFACH and PEZCA for 200 to 1000 nodes with an interval of 200. It is depicted for energy with $0.8 \mathrm{~J}$. It shows that the proposed ECHO-BAT takes the least time to process followed by LEACH, PEZCA and CFACH.

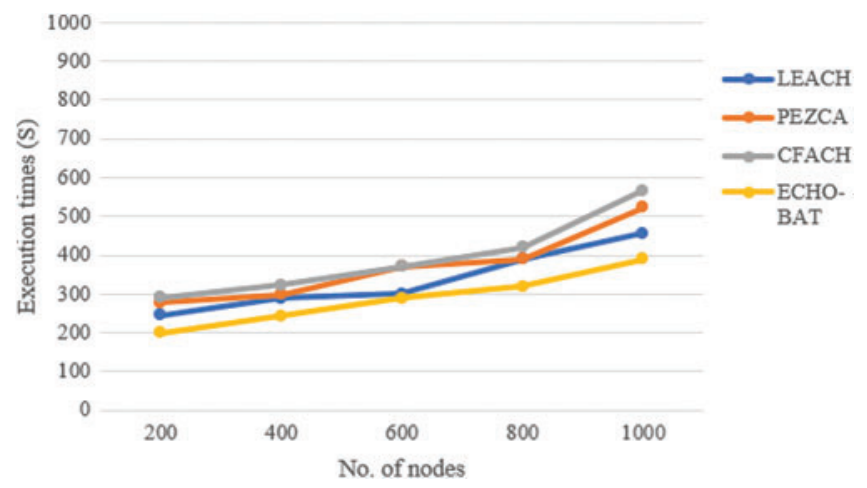

Figure 9: Execution time with $0.7 \mathrm{~J}$

Fig. 10 depicts the processing time taken by ECHO-BAT, LEACH, PEZCA and CFACH with 08.J of energy for 200, 400, 600, 800 and 1000 nodes. It is inferred from Figs. 7-10 that the proposed ECHO-BAT take less time for sending large data with energy level from $0.5 \mathrm{~J}$ through $0.8 \mathrm{~J}$. This experiment is carried out for 1000 nodes. ECHO-BAT is followed by LEACH with the second least execution time.

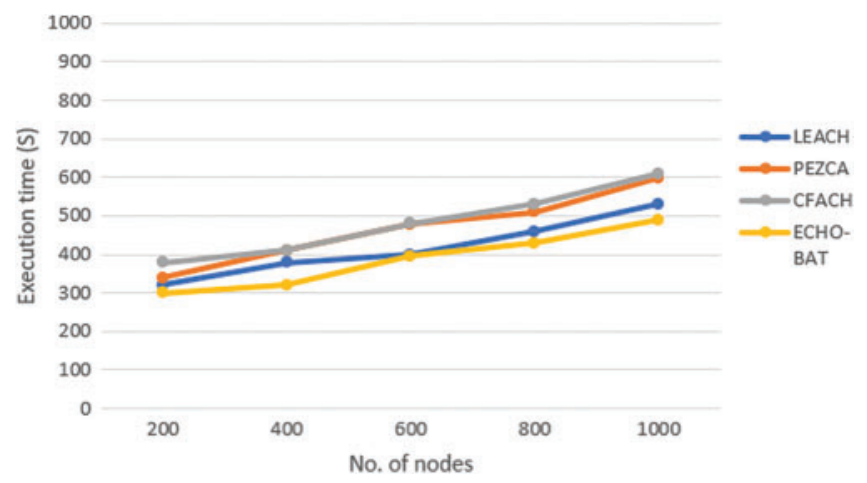

Figure 10: Execution time with $0.8 \mathrm{~J}$

\section{Conclusion}

The lifetime of the wireless sensor networks is a significant factor to be considered to create a stable network. This confront becomes very important in large scale networks where more energy is utilized for packet transmission. Energy consumption in WSNs must be considered which is tedious in creating the network topology. Entropy value and ECHO Location based BAT algorithm (ECHOBAT) is employed to cluster the sensor nodes and to choose the optimal cluster head to communicate with base station. This paper presented an energy efficient model in WSN for 1000 sensor nodes. Proposed ECHO-BAT involves two phases. The first phase is initialization phase and second phase is transmission phase. Initialization works in two steps. Step 1 and 2 deals in choosing the tentative and permanent cluster head using entropy value and Metaheuristic BAT algorithm. Second phase dealt 
with finding an optimal path from each cluster to send and transmit data from cluster heads to base station using Dijkstra algorithm. The effectiveness and the efficiency of the proposed ECHO-BAT are exhibited with the help of simulated results. ECHO-BAT seems to outperform LEACH, PEZCA and $\mathrm{CFACH}$ in terms of network lifetime, death of 1st, 125th and last node, execution time and throughput of the network. The execution time in ECHO-BAT for 200 nodes is $100 \mathrm{~s}$ lesser than other existing methods. Similarly, execution time in ECHO-BAT for 1000 nodes is approximately $150 \mathrm{~s}$ lesser than $\mathrm{LEACH}, \mathrm{PEZCA}$ and $\mathrm{CFACH}$. Identifying the number of clusters in cluster formation and the finding an optimal path depending on the location of base station can be handled in future research work.

Acknowledgement: This work was supported by Taif University Researchers Supporting Program (project number: TURSP-2020/195), Taif University, Saudi Arabia.

Funding Statement: The authors extend their appreciation to the Deanship of Scientific Research at King Khalid University for funding this work under Grant Number (RGP 1/279/42). www.kku.edu. sa.

Conflicts of Interest: The authors declare that they have no conflicts of interest to report regarding the present study.

\section{References}

[1] M. Eftekhari, E. Kranakis, D. Krizanc, O. MoralesPonce, L. Narayanan et al., "Distributed algorithms for barrier coverage using relocatable sensors," Distributed Computing, vol. 29, no. 5, pp. 361-376, 2016.

[2] A. I. Ferreiro, M. Rabaçal and M. Costa, "A combined genetic algorithm and least squares fitting procedure for the estimation of the kinetic parameters of the pyrolysis of agricultural residues," Energy Conversion and Management, vol. 125, pp. 290-300, 2016.

[3] O. Cheikhrouhou, A. Koubâa and A. Zarrad, "A cloud based disaster management system," Journal of Sensor and Actuator Networks, vol. 9, no. 1, pp. 1-6, 2020.

[4] M. S. Mozamir, R. B. A. Bakar, W. I. S. W. Din and Z. Musa, "GBLN-PSO algorithm for indoor localization in wireless sensor network," in Proc. IOP Conf. Series, vol. 769, no. 1, Chennai, India, IOP Publishing, pp. 12033, 2020.

[5] B. Baranidharan, S. Srividhya and B. Santhi, "Energy efficient hierarchical unequal clustering in wireless sensor networks," Indian Journal of Science and Technology, vol. 7, no. 3, pp. 301, 2014.

[6] Y. H. Robinson and M. Rajaram, "Energy-aware multipath routing scheme based on particle swarm optimization in mobile ad hoc networks," The Scientific World Journal, vol. 2015, pp. 1-9, 2015.

[7] N. A. Alrajeh and J. Lloret, "Intrusion detection systems based on artificial intelligence techniques in wireless sensor networks," International Journal of Distributed Sensor Networks, vol. 9, no. 10, pp. 351047, 2013.

[8] F. Wang and J. Liu, "Networked wireless sensor data collection: Issues, challenges, and approaches," IEEE Communications Surveys \& Tutorials, vol. 13, no. 4, pp. 673-687, 2010.

[9] P. Rawat, S. Chauhan and R. Priyadarshi, "Energy-efficient clusterhead selection scheme in heterogeneous wireless sensor network," Journal of Circuits, Systems and Computers, vol. 29, no. 13, pp. 2050204, 2020.

[10] W. Osamy, A. Salim and A. M. Khedr, "An information entropy based-clustering algorithm for heterogeneous wireless sensor networks," Wireless Networks, vol. 26, no. 3, pp. 1869-1886, 2020.

[11] M. Shanmugam and A. Ramasamy, "Sensor-based turmeric finger growth characteristics monitoring using embedded system under soil," International Journal of Distributed Sensor Networks, vol. 10, no. 6, pp. 476176, 2014.

[12] J. Pradeep and M. P. M. Kumar, "Distributed entropy energy-efficient clustering algorithm for heterogeneous wireless sensor network based chaotic firefly algorithm cluster head selection," Journal of Critical Reviews, vol. 7, no. 8, pp. 1208-1215, 2020. 
[13] D. Gong, Y. Yang and Z. Pan, "Energy-efficient clustering in lossy wireless sensor networks," Journal of Parallel and Distributed Computing, vol. 73, no. 9, pp. 1323-1336, 2013.

[14] P. Kuila and P. K. Jana, "A novel differential evolution based clustering algorithm for wireless sensor networks," Applied Soft Computing, vol. 25, pp. 414-425, 2014.

[15] L. Qing, Q. Zhu and M. Wang, "Design of a distributed energy-efficient clustering algorithm for heterogeneous wireless sensor networks," Computer Communications, vol. 29, no. 12, pp. 2230-2237, 2006.

[16] M. Ye, C. Li, G. Chen and J. Wu, "EECS: An energy efficient clustering scheme in wireless sensor networks," in Proc. Int. Performance Computing and Communications Conf. (IPCCC), Phoenix, USA, pp. 535-540, 2005.

[17] Z. Liu, Q. Zheng, L. Xue and X. Guan, "A distributed energy-efficient clustering algorithm with improved coverage in wireless sensor networks," Future Generation Computer Systems, vol. 28, no. 5, pp. 780-790, 2012.

[18] W. Yang and W. T. Zhu, "Voting-on-grid clustering for secure localization in wireless sensor networks," in Proc. Int. Conf. on Communications (ICC), Cape Town, South Africa, pp. 1-5, 2010.

[19] X. Cao, H. Zhang, J. Shi and G. Cui, "Cluster heads election analysis for multi-hop wireless sensor networks based on weighted graph and particle swarm optimization," in Proc. Int. Conf. on Computing, Networking and Communications (ICNC), vol. 7, Jinan, China, pp. 599-603, 2008.

[20] S. A. Sert, H. Bagci and A. Yazici, "MOFCA: Multi-objective fuzzy clustering algorithm for wireless sensor networks," Applied Soft Computing, vol. 30, pp. 151-165, 2015.

[21] H. Taheri, P. Neamatollahi, O. M. Younis, S. Naghibzadeh and M. H. Yaghmaee, "An energy-aware distributed clustering protocol in wireless sensor networks using fuzzy logic," Ad Hoc Networks, vol. 10, no. 7, pp. 1469-1481, 2012.

[22] J. Kim, D. Lee, J. Hwang, S. Hong, D. Shin et al., "Wireless sensor network (wsn) configuration method to increase node energy efficiency through clustering and location information," Symmetry, vol. 13, no. 3, pp. 390, 2021.

[23] T. Qiang, W. Bingwen and D. Zhicheng, "MS-Leach: A routing protocol combining multi-hop transmissions and single-hop transmissions," in Proc. Pacific-Asia Conf. on Circuits, Communications and Systems (PACCS), Chengdu, China, pp. 107-110, 2009.

[24] M. Barbehenn, "A note on the complexity of dijkstra's algorithm for graphs with weighted vertices," IEEE Transactions on Computers, vol. 47, no. 2, pp. 262-263, 1998.

[25] M. N. Halgamuge, M. Zukerman, K. Ramamohanarao and H. L. Vu, "An estimation of sensor energy consumption,” Progress in Electromagnetics Research B, vol. 12, pp. 259-295, 2009. 